\title{
Nonlinear Evolution and Breakdown of Azimuthally Compact Crossflow Vortex Pattern over a Yawed Cone
}

\author{
Meelan Choudhari, ${ }^{*}$ Fei Li, ${ }^{\dagger}$ and Pedro Paredes ${ }^{\ddagger}$ \\ NASA Langley Research Center, Hampton, VA, 23681 \\ Lian Duan ${ }^{\S}$ \\ Missouri University of Science and Technology, Rolla, MO 65409
}

\begin{abstract}
Hypersonic boundary-layer flows over a circular cone at moderate angle of incidence can support strong crossflow instability in between the windward and leeward rays on the plane of symmetry. Due to the more efficient excitation of stationary crossflow vortices by surface roughness, a possible path to transition in such flows corresponds to rapid amplification of the high-frequency secondary instabilities of finite amplitude stationary crossflow vortices. In the present paper, the previous analyses of crossflow instability over a 7degree half-angle, yawed circular cone in a Mach 6 free stream have been extended to the nonlinear evolution of azimuthally localized crossflow vortex packets and the amplification characteristics and nonlinear breakdown of high-frequency secondary instabilities associated with those packets. A comparison between plane marching PSE and direct Navier-Stokes simulations (DNS) reveals favorable agreement in regard to mode shapes, most amplified disturbance frequencies, and $\mathrm{N}$-factor evolution. In contrast, the quasiparallel predictions are found to result in severe underprediction of the $\mathbf{N}$-factors. The direct numerical simulations also indicate that the breakdown of secondary instabilities in a 3D hypersonic boundary layer shares certain common features with the previous computations of crossflow transition over subsonic swept wings.
\end{abstract}

\section{Nomenclature}

$f \quad=$ frequency of instability waves $[\mathrm{kHz}]$

$M_{\infty}=$ freestream Mach number (nondimensional)

$N=$ logarithmic amplification ratio of a fixed frequency secondary instability mode (nondimensional)

$N_{k e}=\mathrm{N}$-factor based on disturbance kinetic energy integrated across the cross-section (nondimensional)

$N_{u}=\mathrm{N}$-factor based on peak $u$-velocity fluctuation (nondimensional)

$n=$ azimuthal wavenumber, i.e., number of waves across circumference (nondimensional)

$p^{\prime} \quad=$ pressure fluctuation $[\mathrm{Pa}]$

$P_{\infty}=$ freestream pressure $[\mathrm{Pa}]$

$q_{w}=$ surface heat flux $\left[\mathrm{W} / \mathrm{m}^{2}\right]$

$R e=$ freestream unit Reynolds number $\left[\mathrm{m}^{-1}\right]$

$t=$ time [sec]

$T_{w} \quad=$ wall temperature $[\mathrm{K}]$

$T_{\infty}=$ freestream temperature $[\mathrm{K}]$

$u=$ streamwise velocity $[\mathrm{m} / \mathrm{s}]$

$u^{\prime}=$ fluctuation in streamwise velocity $[\mathrm{m} / \mathrm{s}]$

$u_{r m s}=$ root mean square fluctuation in streamwise velocity $[\mathrm{m} / \mathrm{s}]$

$U_{\infty}=$ freestream velocity $[\mathrm{m} / \mathrm{s}]$

$X=$ axial coordinate $[\mathrm{m}]$

$Y=$ wall-normal distance $[\mathrm{m}]$

$\alpha=$ angle of attack [deg]

$\phi \quad=$ azimuthal coordinate with respect to windward meridian [deg]

\footnotetext{
*Aerospace Technologist, Meelan.M.Choudhari@nasa.gov, Associate Fellow, AIAA.

${ }^{\dagger}$ Aerospace Technologist, Fei.Li@nasa.gov

‡NASA NPP Fellow, pedro.paredesgonzalez@nasa.gov, Member, AIAA.

§Assistant Professor, duanl@mst.edu, Senior Member, AIAA.
} 


\section{Introduction}

$\mathrm{B}$ oundary layer transition from the laminar to a turbulent state is known to have a major impact on the design and performance of hypersonic flight vehicles. When the vehicle surface is relatively smooth, the transition process is initiated by linear instabilities of the laminar boundary layer, including second (or Mack) mode instability, Görtler instabilities, stationary and traveling modes of crossflow instability, and the attachment line instability. ${ }^{1-3}$

Regardless of the speed regime, linear stability correlations have been quite successful in predicting the onset of transition when a single instability mechanism dominates the transition process. However, measurements of crossflow instability in low-speed boundary layers have exposed the shortcomings of applying purely linear predictive models to transition in 3D boundary layers by revealing the importance of nonlinear effects during crossflow dominated transition. ${ }^{3}$ The canonical configuration of a circular cone at angle of attack (AOA) includes the necessary elements to study both mixed mode transition and crossflow development in the context of both supersonic $^{4-12}$ and hypersonic ${ }^{13-32}$ boundary-layer flows. Instability mechanisms for the boundary-layer flow over an elliptic cone have been investigated in Refs. [19], [29], and [33]-[47]. While the elliptic cone configuration supports similar instability mechanisms as the circular cone at an angle of incidence, the details of transition patterns can be different as noted in Ref. [42].

Experimental measurements of traveling crossflow instability on an elliptic cone ${ }^{47}$ have yielded encouraging comparison with the linear stability predictions by Li et al. ${ }^{19}$ Measurements of crossflow instability over a yawed circular cone in three different Mach 6 wind tunnels have been reported in Refs. [20-25]. Besides a low frequency peak between 15 to $60 \mathrm{kHz}$ that is presumably related to traveling crossflow disturbances, the circular cone measurements from Refs. [20-23] indicate higher frequency peaks, particularly in the vicinity of 300 to $350 \mathrm{kHz}$. Measurements by Craig and Saric ${ }^{24-25}$ revealed lower frequency disturbances near $110 \mathrm{kHz}$ that were still higher than the traveling crossflow disturbances of the unperturbed boundary layer.

Computations of secondary instability of crossflow modes in a hypersonic boundary layer were first reported by Li et al. ${ }^{29}$ The predicted secondary instabilities with the highest growth rates had frequencies that were comparable to those measured in the experiments at the Technical University of Braunschweig ${ }^{20}$ and the Purdue University, ${ }^{21-23}$ but the computations had also revealed the existence of less unstable modes with lower disturbance frequencies that were comparable to those measured by Craig and Saric. ${ }^{25}$ Recently, more detailed secondary instability analyses for large amplitude stationary crossflow vortices over the yawed cone were described by Li et al. ${ }^{30,31}$ and Moyes et al. ${ }^{32}$ These works identified three major types of instability modes: those that originate from low-frequency traveling crossflow modes and high-frequency Mack mode instabilities of the unperturbed boundary layer, but get modulated (and potentially further destabilized) by the presence of large amplitude stationary crossflow vortices; and genuine, high-frequency secondary instability modes that arise entirely due to the presence of large amplitude stationary crossflow vortices and are concentrated in the shear layer that bounds those vortices. Similar to Li et al., ${ }^{30,31}$ this paper will focus on all high-frequency secondary instabilities, including those that originate from the Mack modes and those that emerge only when the stationary vortices become sufficiently strong.

Choudhari et al. ${ }^{48}$ reported computations of azimuthally compact, stationary crossflow vortex packets over a 7degree half-angle, yawed circular cone in a Mach 6 free stream, namely, stationary crossflow vortex patterns generated by azimuthally localized roughness height distributions and the breakdown characteristics of highfrequency secondary instabilities associated with those crossflow vortex packets. Such analysis is important for both predicting and controlling laminar-turbulent transition over the cone, since roughness elements provide a potential means to control the transition process initiated by stationary crossflow vortices. The present paper represents a continuation of that research, wherein we continue to address the effects of crossflow vortex modulation due to combined azimuthal inhomogeneity of the unperturbed boundary layer over a yawed cone and of the roughness height distribution over the cone surface. Specifically, the findings from Ref. [48] are extended in two important aspects: (i) nonparallel predictions for the evolution of secondary instabilities in the azimuthally inhomogeneous, three-dimensional boundary layer and a comparison between those predictions and DNS results, and (ii) the effects of nonlinearity on the development of those secondary instabilities.

The remaining sections of this paper are laid out as follows. A brief summary of the flow configuration of interest is given in Section II, which also describes the analysis codes used in this study. The generation of stationary crossflow vortices via selected azimuthal distributions of roughness elements and the subsequent evolution the generated vortices is considered in Section III. Evolution of the secondary instabilities sustained by finite amplitude, stationary crossflow vortices is outlined in Section IV, which also includes a comparison between the DNS results from Ref. [48] and the predictions of parabolized stability equations (PSE). Preliminary results 
pertaining to the nonlinear evolution of the dominant family of secondary instability modes is investigated in Section V. Summary and concluding remarks are presented in Section VI.

\section{Flow Configuration and Analysis Codes}

The flow configuration of interest corresponds to a circular cone at angle of attack to a uniform, hypersonic free stream. The primary configuration for the Purdue experiments corresponds to a 0.457 meter $(18 \mathrm{inch})$ long, nominally sharp nosed cone (modeled with a nose radius of $10^{-4}$ times the cone length) with a half angle of 7 degrees and an angle of attack, $\alpha$, equal to 6 degrees. The freestream conditions correspond to a free-stream Mach number of $M_{\infty}=6$, unit Reynolds number $R e=10.8 \times 10^{6}$ per meter, and free-stream temperature $T_{\infty}=52.44 \mathrm{~K}$. The freestream velocity at these conditions corresponds to $U_{\infty}=870.9 \mathrm{~m} / \mathrm{s}$. The temperature of the model surface, $T_{w}$, is equal to $300 \mathrm{~K}$. The above flow configuration is identical to that used by Li et al. ${ }^{30,31}$ and the same computational mesh and basic state solution from those studies are used in the present work as well. For the sake of completeness, the details of the computational scheme are repeated below.

The unperturbed boundary-layer flow over the cone is computed using the VULCAN (Viscous Upwind aLgorithm for Complex flow ANalysis) software. ${ }^{49}$ The code solves the unsteady, conservation equations appropriate for laminar or turbulent flow of calorically or thermally perfect gases with a spatially second order accurate cell-centered finite volume scheme. In the present computations, the inviscid fluxes were constructed using the MUSCL $\kappa=0$ scheme, the van Albada gradient limiter ${ }^{50}$ and the Low Dissipation Flux Split Scheme (LDFSS) of Edwards. ${ }^{51,52}$ The cell face gradients required to construct the viscous fluxes were obtained using an auxiliary control volume approach that results in a compact viscous stencil that produces a second order accurate approximation of the full Navier-Stokes viscous fluxes. In previous work, similar computations of the mean flow over an elliptic cone configuration were cross-validated against the solutions obtained with the LAURA code ${ }^{53}$ for various grid sizes. ${ }^{20}$ Additional grid convergence analysis for the present flow configuration is reported by Li et al. ${ }^{31}$

The evolution of stationary crossflow disturbances in the boundary-layer flow is computed by using the VULCAN solution described above as the basic state. The stationary crossflow vortices are excited via an array of axially localized roughness elements centered at $X=0.18 \mathrm{~m}$. The streamwise shape of the roughness elements corresponds to a half-wavelength cosine shape with an axial wavelength of $0.008 \mathrm{~m}$ and a peak height perturbation of $5 \mu \mathrm{m}$. Different azimuthal distributions of roughness height are used, including an azimuthally periodic forcing with a single azimuthal wavenumber of $n=60$ (where $n$ denotes the number of vortex wavelengths across the full circumference of the circular cone) and various localized azimuthal distributions obtained by windowing the periodic distribution over one half wavelength, one wavelength, three wavelengths, and five wavelengths, respectively. The choice of roughness parameters used herein enables one to approximate the observed spacing and the strength of nonlinear crossflow vortices as gauged by the rollup of mass-flux contours across the cross section and the spatial region (axial and azimuthal locations) of vortices with sufficiently large amplitudes.

The working fluid is assumed to be perfect gas (air) and the usual constitutive relations for a Newtonian fluid are used: the viscous stress tensor is linearly related to the rate-of-strain tensor, and the heat flux vector is linearly related to the temperature gradient through Fourier's law. The coefficient of viscosity is computed from Sutherlands's law, and the coefficient of thermal conductivity is computed by assuming a constant Prandtl number $\mathrm{Pr}=0.71$. A detailed description of the governing equations and their numerical solution is given by $\mathrm{Wu}$ et al. ${ }^{54} \mathrm{The}$ inviscid fluxes from the governing equations are computed using a seventh-order weighted essentially nonoscillatory finite-difference WENO scheme introduced by Jiang and Shu; ${ }^{55}$ the present scheme also allows the use of limiters ${ }^{54,56}$ that have been optimized to reduce the numerical dissipation. Both an absolute limiter on the WENO smoothness measurement and a relative limiter on the total variation are employed simultaneously during the simulation. The viscous fluxes are discretized using a fourth-order central difference scheme and time integration is performed using a third-order low-storage Runge-Kutta scheme. ${ }^{57}$ The numerical code has been previously applied to simulation of turbulence in hypersonic boundary layers ${ }^{58}$ as well as laminar-turbulent transition due to crossflow instability in swept wing boundary layers. ${ }^{59,60}$ The lessons learned from these simulations were applied to develop the computational grid for stationary crossflow evolution in the present work.

The primary instability computation is performed on a grid consisting of $600 \times 1200 \times 140$ points in the axial $(X)$, azimuthal $(\phi)$, and wall-normal $(Y)$ directions, respectively. Without any loss of generality, the stationary crossflow disturbances are assumed to be symmetric about the windward and leeward planes, so that only one half of the circular cone was included in the computational domain. The grid points are spaced uniformly in the azimuthal direction. For select cases, the numerical accuracy of the basic state calculation was verified by by Li et al. ${ }^{30,31}$ via 
computations on a finer grid with significantly higher resolution in the azimuthal and radial directions. Additional calculations for similar configurations had also established that the grid spacing in the axial direction is adequate for the purpose of computing the evolution of the crossflow vortices.

In a precursor to this paper, Choudhari et al ${ }^{48}$ investigated the secondary instability of the finite amplitude stationary crossflow vortices emanating from an azimuthally compact source by performing quasiparallel, spatial predictions based on planar eigenvalue analysis, as previously described by Li and Choudhari, ${ }^{61,62}$ for swept wing boundary layers. For an azimuthally periodic source that leads to a quasiperiodic pattern of crossflow vortices in the downstream region, Li et al. ${ }^{30,31}$ had employed both quasiparallel predictions and a nonparallel framework based on plane marching parabolized stability equations (PSE) as described by Paredes et al. ${ }^{63,64}$ For cases involving a quasiperiodic vortex behavior (which applies both when a periodic roughness array extends around the entire circumference of the cone and to the vortex/vortices near the center of a sufficiently wide vortex pattern in cases where the periodic roughness array is truncated to a noncompact azimuthal width), the basic state for each individual vortex can be made azimuthally periodic via a combination of detrending and Fourier low-pass filtering and then used for local secondary instability computations. For further details of the procedure used for secondary instability analysis in this case, the reader is referred to the earlier works by Li et al ${ }^{30,31}$ In the opposite limiting case that corresponds to a sufficiently compact vortex pattern (i.e., where the entire pattern is limited to a small number of vortices that are nearly parallel to each other), the basic state for secondary instability must include the entire pattern at a given axial location. Results of this type were presented by Choudhari et al., ${ }^{48}$ where limited comparisons were made between the predictions of quasiparallel secondary instability theory and direct numerical simulation (DNS) of high frequency secondary disturbances that were forced through small amplitude suction and blowing at the cone surface. Those comparisons are extended in the present paper to include nonparallel predictions based on plane marching PSE.

Since the boundary layer flow modified by the crossflow vortices already exhibits short-scale azimuthal variations, the unsteady forcing in the DNS of Choudhari et al. ${ }^{48}$ was chosen to be invariant in the azimuthal direction and localized at the same location as the array of roughness elements. The time dependence of the forcing function includes a superposition of time harmonic signals corresponding to multiple frequencies from $20 \mathrm{kHz}$ to $480 \mathrm{kHz}$. Fourier decomposition of the output signal is used to analyze the disturbance evolution at each selected frequency. In the present paper, we pursue an alternate strategy of initiating high-frequency instabilities at a single fixed frequency of $f=240 \mathrm{kHz}$ at the inflow boundary. The azimuthal and wall-normal distributions of the inflow disturbance field correspond to the mode shape predicted by plane marching PSE. Additional calculations have also been performed by employing the wall-forcing strategy from Ref. [48], wherein larger forcing amplitudes are now used to allow the secondary instabilities to reach nonlinear amplitude levels within the length of the computational domain and potentially induce an onset of laminar-turbulent transition. The latter simulation involves a significantly finer grid than the grids used to compute the stationary crossflow evolution. The grid spacings in this case were based on similar computations of swept wing transition ${ }^{60}$ with the same flow solver and due care was taken to ensure that the grid spacing in viscous wall units are also adequate to resolve the late stage transition and the fully turbulent dynamics.

\section{Effect of Azimuthal Distribution of Roughness Height}

Computational results pertaining to stationary crossflow vortices in the boundary-layer flow over the cone are presented in this section. As mentioned previously, the evolution of the quasiperiodic pattern of stationary crossflow vortices due to an azimuthally periodic array of roughness elements was described by Li et al. ${ }^{31}$ Vortex patterns due to azimuthally compact distributions of roughness elements were investigated by Choudhari et al. ${ }^{48}$ In this paper, the latter results are extended to include additional roughness configurations that bridge the gap between the previously examined limiting cases of azimuthally periodic and azimuthally compact forcing, respectively.

The periodic forcing case corresponds to a forcing wavenumber of $n=60$. The latter wavenumber was chosen to approximate the azimuthal wavelength of the crossflow modes found in the experiments of Craig and Saric. ${ }^{25}$ Farther downstream from the forcing location, the vortex structures, or equivalently, streaks generated by the roughness pattern move toward the leeward ray and the streak spacing changes as a result of the nonparallel-flow effects. Consequently, there is a pronounced difference between the larger vortex wavelength near the sideline ray and the smaller wavelength on the leeward side.

The effect of the azimuthal extent of the roughness height distribution on the generated vortex patterns is shown in Fig. 1, which shows the axial velocity contours at $X=0.4 \mathrm{~m}$ for various selected roughness element distributions. Figure 1(a) corresponds to the periodic forcing case, ${ }^{31}$ whereas Figs. 1(d) and 1(e) correspond to azimuthally compact forcing where the periodic forcing is truncated to a single azimuthal wavelength and half azimuthal 
wavelength, respectively. The half wavelength azimuthal distribution resembles a single protuberance, whereas the single wavelength distribution involves a protuberance and a dimple adjacent to each other. These two cases were previously described in Ref. [48]. Here, we include additional results corresponding to intermediate azimuthal extents of the forcing, equal to five (Fig. 1(b)) and three (Fig. 1(c)) azimuthal wavelengths, respectively.

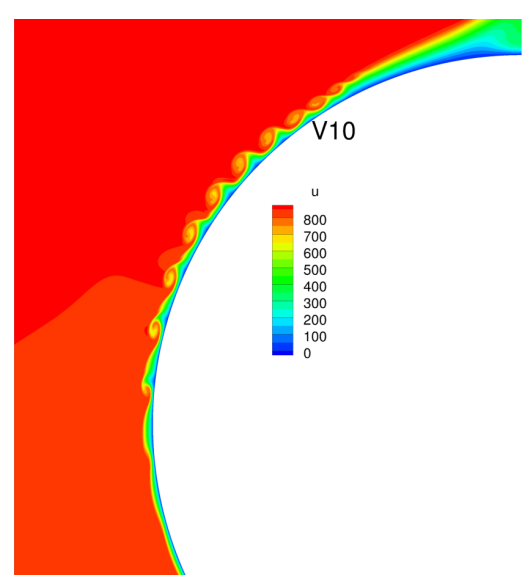

(a) Periodic forcing

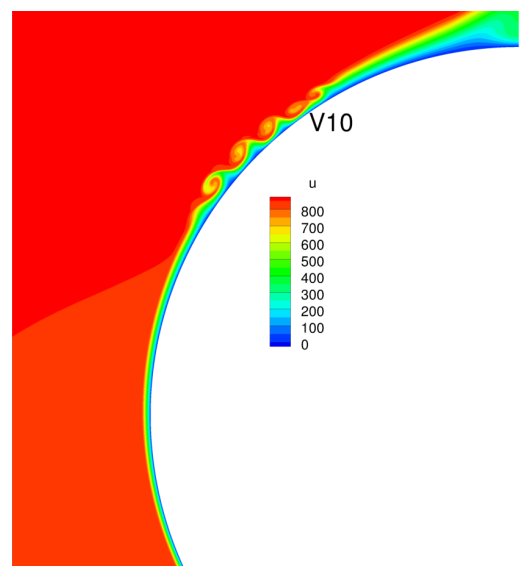

(c) 3-wavelength forcing

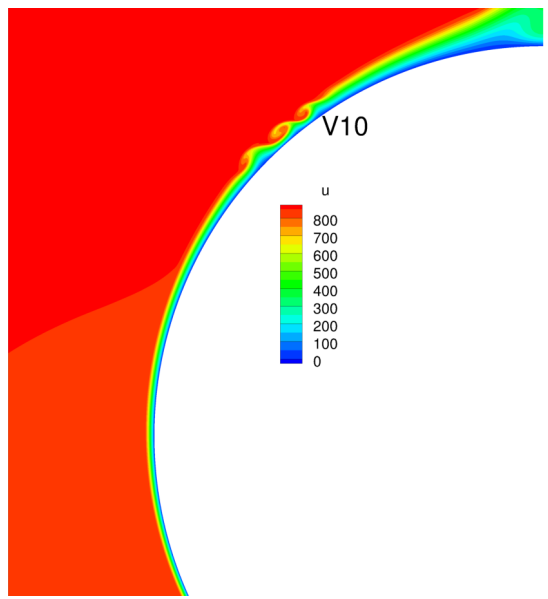

(e) Half wavelength forcing

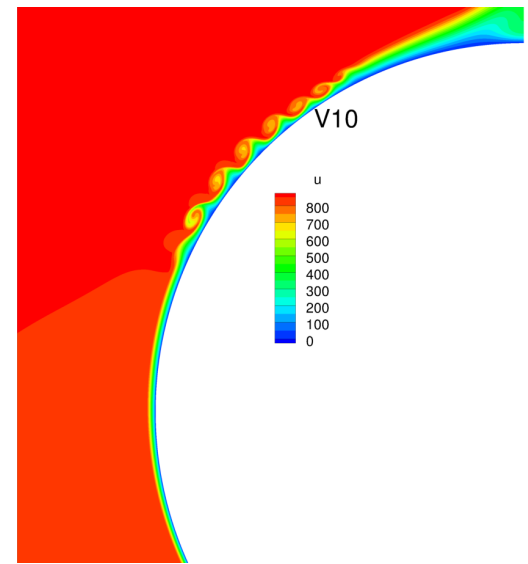

(b) 5-wavelength forcing

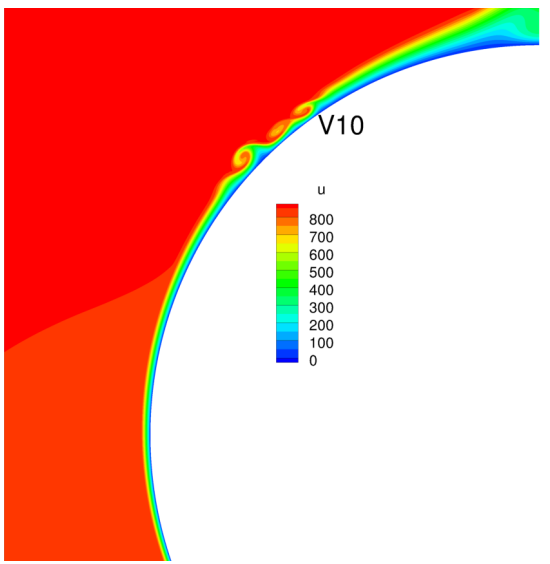

(d) Single wavelength forcing

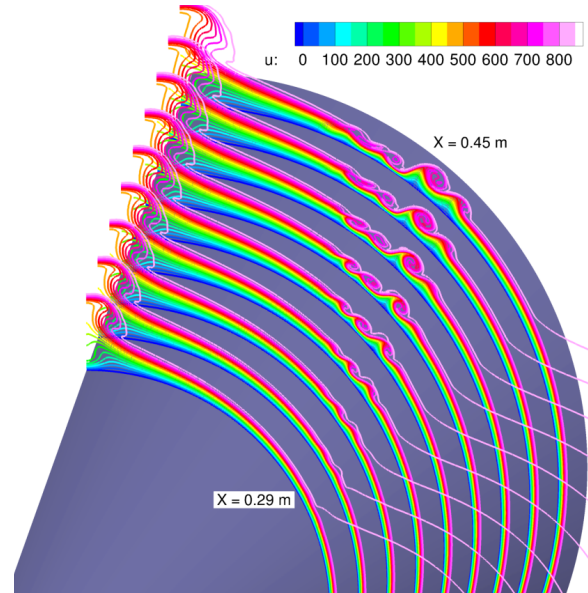

(f) Multiple axial stations for the case with single wavelength forcing (mirror image of vortex pattern from part (b)). Distance between each pair of adjacent stations is $0.02 \mathrm{~m}$.

Fig. 1. Axial velocity contours at $X=0.4 \mathrm{~m}$ for roughness distribution with varying azimuthal extent. 
As expected, the localized distributions generate considerably narrower crossflow vortex patterns (Figs 1(b)(e)) compared to the vortex pattern in the periodic case (Fig. 1(a)). Only three dominant vortex structures are observed for each of the two localized distributions. The azimuthal location of roughness elements in the latter two cases was chosen such that the three dominant vortices excited by the azimuthally localized roughness elements approximately align with vortices 8 through 10 from the vortex pattern excited by the periodic roughness height distribution. ${ }^{31}$ The location of vortex 10 from the periodic roughness case is labeled in Fig. 1(a), and for reference, is also indicated in the remaining parts of Fig. 1. For each of the roughness configurations considered in Fig. 1, the velocity contours associated with the dominant vortices exhibit strong roll-up as reflected in the overturned contours at $X=0.4 \mathrm{~m}$, indicating a highly nonlinear crossflow disturbance field at that location. The number of visibly prominent vortices within the overall pattern progressively decreases from 12 in the periodic case (Fig. 1(a)) to three in the half-wavelength case (Fig. 1(e)). As the azimuthal extent of the forcing is increased, the case of three wavelength forcing is the first to show an emergence of quasiperiodic behavior for the center vortices (Fig. 1(c)) and that quasiperiodic nature becomes progressively more prominent in the cases corresponding to five wavelength forcing (Fig. 1(b)) and a periodic roughness array (Fig. 1(a)), respectively. The similarity of the dominant vortex evolution in the three wavelength and five wavelength cases with that in the periodic-forcing case would become further apparent from the subsequent comparison between the vortex amplitudes.

The trajectories corresponding to the centerlines of selected stationary vortices from the various cases in Fig. 1 are shown in Fig. 2(a). Specifically, the figure shows the four vortices detected within the compact vortex patterns of Figs. 1(d) and 1(e), as well as an additional, (i.e., fifth) inboard vortex from the other three cases. The extra vortices seen in Figs. 1(a) through 1(c) are not included in this figure. The ordinate in Fig. 2(a) denotes the azimuthal angle $\phi$ with respect to the windward plane, which increases to 180 degrees at the leeward plane. Figure 2(a) shows that the vortex trajectories for all five roughness distributions align very well with each other, except for some small discrepancies in the downstream portions of the trajectories $(X>0.35 \mathrm{~m})$ that are likely to be associated with nonlinear effects at higher vortex amplitudes. The overall comparison suggests that the vortex trajectories are insensitive to the azimuthal extent of the forcing, at least for the cases displayed in Fig. 1.

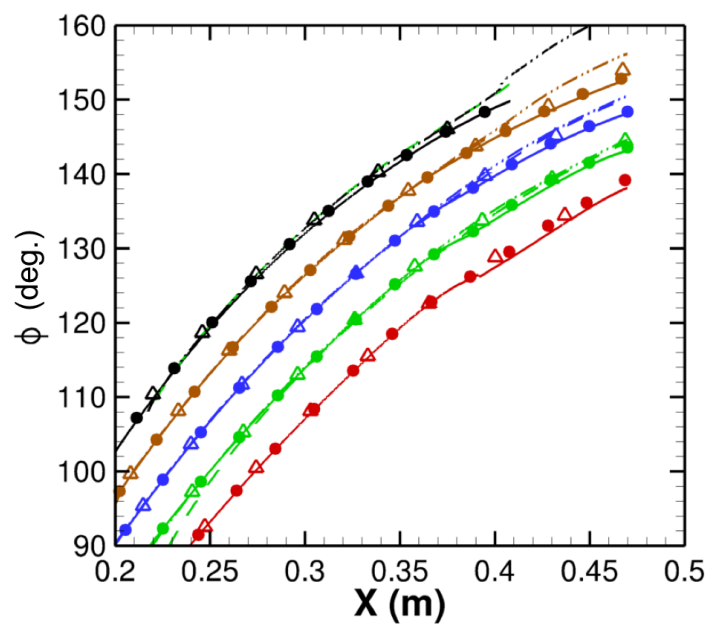

(a) Vortex trajectories. Colors distinguish between five different vortices from the vortex patterns.

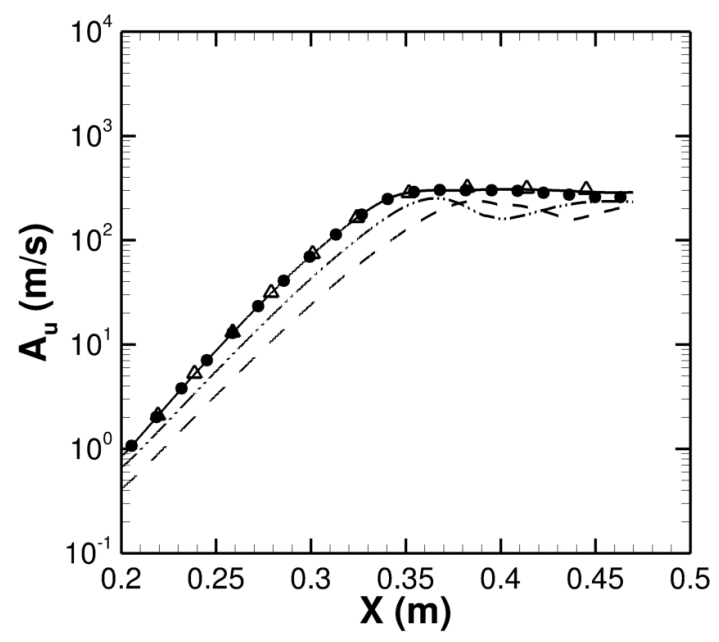

(b) Peak vortex amplitude (corresponding to vortex trajectory in blue from art (a) of the figure) as measured by maximum velocity perturbation associated with each forcing distribution.

Fig. 2. Comparison of vortex characteristics associated with different azimuthal distributions of roughness height. The line/symbol types indicate azimuthal extent of roughness distribution (solid: periodic, dashed: half wavelength, dash-dot: single wavelength, filled circles: three wavelengths, open triangles: five wavelengths).

The amplitude of the dominant crossflow vortex for each of the two compact roughness cases (Figs. 1(d) and 1(e)), along with the amplitude of the corresponding vortex in the other three cases (which corresponds to vortex 8 
from the periodic case of Li et al. ${ }^{31}$ ) are plotted as functions of the axial coordinate in Fig. 2(b). The vortex amplitude $A_{u}$ plotted in this figure corresponds to the peak value of the velocity perturbation parallel to the vortex trajectory at a given axial location. Figure 2(b) shows that the amplitude evolution curves in all cases are nearly parallel to each other until the nonlinear effects come into play. The distances between the parallel evolution curves indicate the influence of the azimuthal distribution of roughness height on the effective initial amplitude of the dominant vortex.

Figure 2(b) also shows that the vortex amplitudes for the three wavelength and five wavelength forcing, respectively, are nearly the same as the vortex amplitude in the periodic-forcing case. As a result of the considerably larger initial amplitude in these cases, the dominant vortex (corresponding to blue trajectories in Fig. 2(a)) achieves nonlinear amplitude levels just upstream of $X=0.35 \mathrm{~m}$ and remains in a quasisaturated state within the downstream portion of the computational domain. The amplitude of the dominant vortex in the single wavelength case reaches its peak value just downstream of $X=0.36 \mathrm{~m}$, and exhibits a weak oscillatory pattern thereafter. This oscillatory evolution of the vortex amplitude is associated with a cyclic energy exchange between the fundamental mode corresponding to the vortex wavelength and the mean flow distortion induced by nonlinear effects. ${ }^{65}$ The vortex amplitude in the half wavelength case displays a similar behavior, except that its evolution lags behind that in the other four cases. The post-saturation vortex amplitudes in the two limiting cases of a compact vortex pattern are similar to each other, but remain lower than the peak vortex amplitude achieved in the remaining three cases with larger extents of roughness height distribution.

\section{High-Frequency Secondary Instabilities of a Compact Vortex Pattern}

In this section, the DNS data from Choudhari et al., ${ }^{48}$ is used to assess the accuracy of secondary instability predictions for a compact vortex pattern due to the single wavelength forcing as shown earlier in Fig. 1(d). The vortex pattern is relatively compact in comparison with the quasiperiodic vortex pattern studied by Li et al., ${ }^{31}$ and hence, its instability characteristics need to be analyzed in the context of the entire vortex pattern rather than on a local basis, either via planar eigenvalue analysis ${ }^{62}$ or by using plane marching PSE. ${ }^{63}$ A limited set of quasiparallel predictions based on planar eigenvalue analysis was presented in Ref. [48]. Here, we use the plane marching PSE to extend those predictions to nonparallel evolution of the secondary instabilities. For an in-depth description of each methodology and the pertinent numerics, the reader is referred to the previous papers. ${ }^{31,62,63}$

\section{A. Nonparallel stability predictions for a compact vortex pattern}

In general, the compact pattern of crossflow vortices can support two different types of unstable, high-frequency, secondary instability modes. One of them originates as unstable Mack modes in the upstream region, where the amplitude of the stationary crossflow pattern is still small, and continues to get amplified as the crossflow vortices gain in strength. Following Li et al., ${ }^{31}$ this family of modes is denoted with the prefix MM in this paper. There exist additional families of unstable modes, but they emerge only after the crossflow vortices have become sufficiently strong. Thus, at the onset of their amplification, the latter modes are equivalent to genuine secondary modes associated with localized instabilities of the shear layer that bounds the crossflow vortex pattern, and hence, these modes are denoted with the suffix SI. As seen later, the MM modes also become concentrated in the shear layer region when the crossflow vortices become strong, but the classification of secondary instabilities as MM or SI modes pertains to the instability mechanism where those modes first become unstable.

For the compact crossflow vortex pattern of interest in this paper, the fluctuations associated with both MM and SI modes extend over a majority of the vortex pattern. However, the peak fluctuations associated with any given mode typically occur inside a single crossflow vortex within the overall pattern. The base flow contours from Fig. 1(f) indicate that there are two prominent crossflow vortices at $X=0.33 \mathrm{~m}$, whereas an additional vortex becomes visible by $X=0.37 \mathrm{~m}$. For simplicity, the dominant vortex on the windward side is denoted as V1 from here on, whereas the adjacent vortex (corresponding to the middle vortex at $X=0.37 \mathrm{~m}$ ) will be denoted as V2 in the remaining paper. The remaining vortices do not play a significant role in the amplification of secondary instabilities.

Both MM and SI modes of secondary instability are subclassified according to whether the peak fluctuations are concentrated in the windward vortex (vortex V1) or in the adjacent vortex V2 toward the leeward plane of symmetry (corresponding to the middle vortex at $X=0.37 \mathrm{~m}$ ). Because the remaining vortices are relatively weak, the peak fluctuations associated with all dominant high-frequency instability modes occur within vortex V1 or vortex V2. Thus, mode MMV1 corresponds to the continuation of the Mack mode that induces peak fluctuations within the vortex V1. As described later, the modal peak may shift to a different vortex in the far downstream region, after the crossflow vortices have reached large amplitudes and begin to interact strongly with each other. However, the 
modal notation adopted herein is based on the location of peak fluctuations within the initial region of instability and that is also representative of a majority of the overall computational domain.

Mode shapes of $\left|u^{\prime}\right|$ fluctuations associated with two representative instability modes predicted by the nonparallel, PSE framework are shown in Figs. 3(a) and 3(b), respectively. Both figures display the crossplane contours of $\left|u^{\prime}\right|$ at $f=240 \mathrm{kHz}$, superposed with gray shade contours of the logarithmic amplification factor (i.e., Nfactor) based on the surface pressure fluctuations in each case. Figure 3(a) shows that, as the amplitude of the crossflow vortices increases from relatively small values near the upstream end of the computational domain to larger, yet modest amplitudes near $X=0.330 \mathrm{~m}$, the location of peak $\left|u^{\prime}\right|$ fluctuations associated with the MMV1 mode moves away from the wall and into the inclined shear layer bounding the stationary crossflow vortex. The $\left|u^{\prime}\right|$ levels in the lower part of the boundary layer are weaker but not negligible. Although not shown, a similar behavior is noted for the MMV2 mode shapes, except that the peak fluctuations are located within the vortex V2. On the other hand, the SI modes tend to be concentrated primarily in the localized shear layers away from the surface as indicated by the SIV2 mode shape in Fig. 3(b). Similar findings were reported by Li et al. ${ }^{31}$ for a quasiperiodic vortex pattern due to an azimuthally periodic source, but they also found certain cases where weak but significant SI mode fluctuations occurred below the peak inside the shear layer.

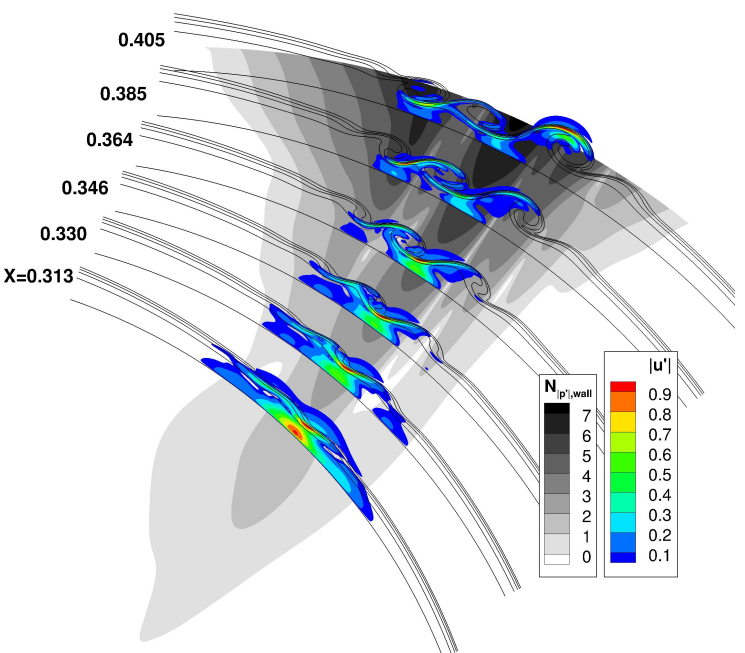

(a) Mode MMV1

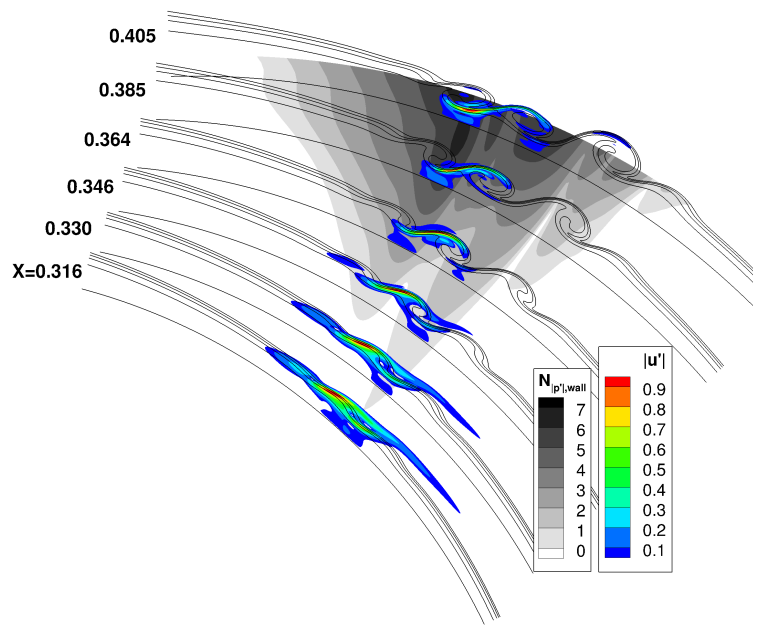

(b) Mode SIV2

Fig. 3. Contours of disturbance amplitudes ("mode shapes") based on $\left|u^{\prime}\right|$ fluctuations at $f=240 \mathrm{kHz}$ and selected axial stations. At each station, the peak fluctuation amplitude is normalized to unity. To highlight the regions of strong fluctuations at multiple axial stations, contours are shown only within the boundary layer region. Gray shaded contours along the cone surface indicate N-factor distribution based on the surface pressure fluctuations.

The axial evolution of the logarithmic amplification ratio, i.e., the $\mathrm{N}$-factor, associated with fixed-frequency secondary disturbances of the MMV1 and MMV2 type are shown in Figs. 4(a) and 4(b), respectively. The N-factors for the SI modes are uniformly lower than those of the MM modes and, for the purpose of illustration, only the results for the SIV2 mode are shown in Fig. 4(c). N-factors for the SIV1 mode are even lower than those of mode SIV2, and hence, they have been omitted from Fig. 4. The MM modes typically display an initial region of growth that is dominated by the Mack mode instability, followed by a downstream region of accelerated growth (manifested via the increased slope of $\mathrm{N}$-factor curves) as the Mack modes get further destabilized by the finite amplitude crossflow vortices. The SI modes owe their amplification entirely to the large amplitudes of the crossflow vortices, and hence, their amplification curves do not include the initial region of growth encountered by the MM modes where those vortices are relatively weak. In the downstream region, where both MM and SI modes coexist, the mode shape and growth rate characteristics of both modes are similar to each other. However, because of the initial phase of growth as predominantly Mack modes of a quasihomogeneous boundary layer, the $\mathrm{N}$-factors for the MM modes are generally higher than those of the SI modes, at least for the range of locations considered in this paper. 


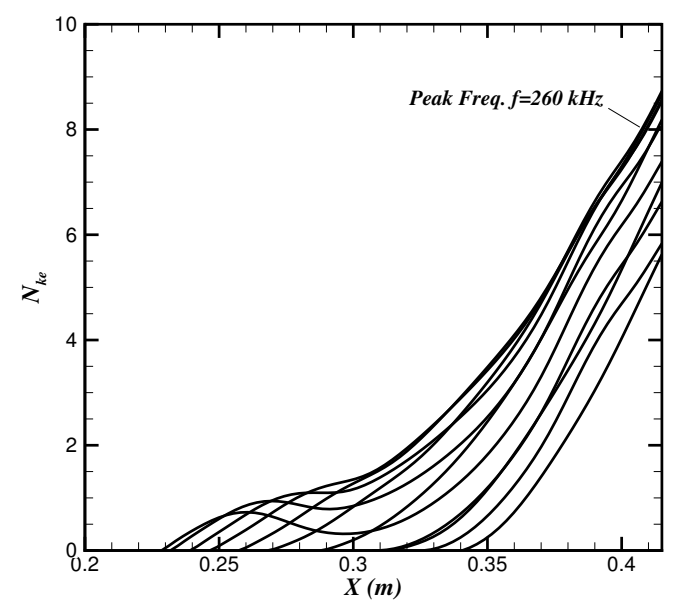

(a) Mode MMV1.

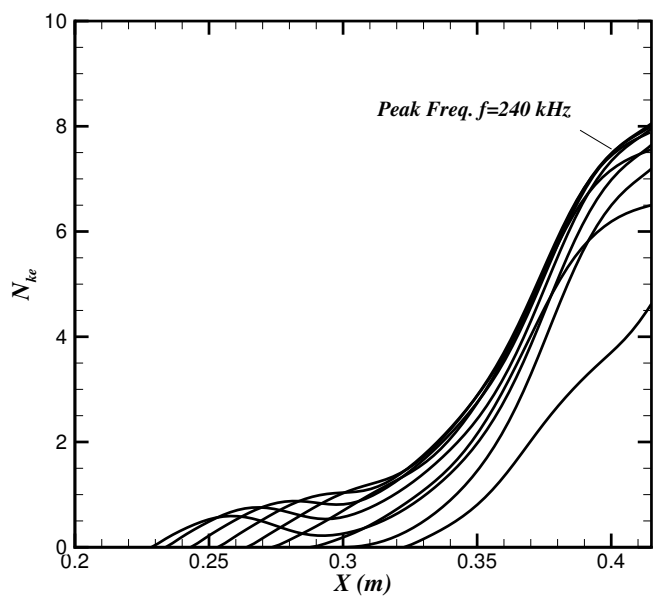

(b) Mode MMV2.

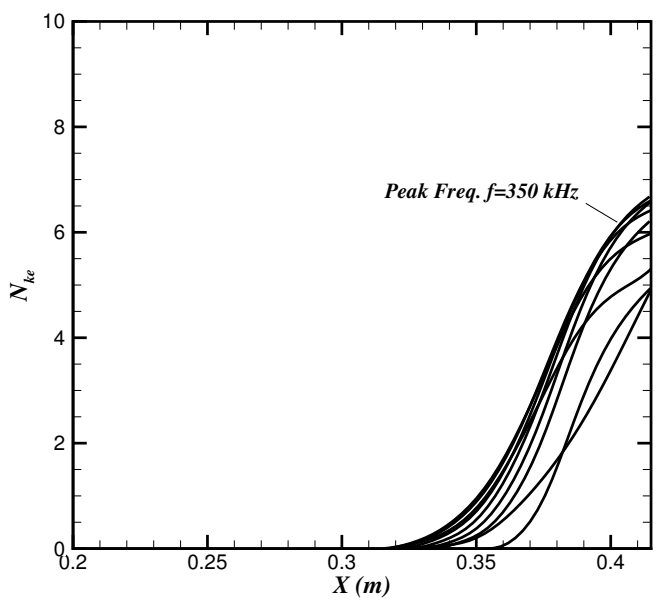

(c) Mode SIV2.

Fig. 4. Amplification characteristics of dominant secondary instability modes of the compact crossflow vortex pattern. Each curve in these figures denotes the $\mathbf{N}$-factor evolution of a fixed frequency disturbance from a specified family of modes.

\section{B. Comparison of disturbance evolution based on DNS and plane marching PSE}

This subsection presents a comparison of the PSE predictions with the DNS results of Choudhari et al. ${ }^{48}$ In those DNS calculations, disturbances at various selected frequencies were introduced via azimuthally uniform, time harmonic, surface suction and blowing over an axially localized strip near $X=0.28 \mathrm{~m}$. The azimuthal grid was clustered within the region of the compact vortex pattern and the axial grid spacing was also reduced near the region of forcing, in order to resolve the receptivity process. Magnitudes of surface pressure fluctuations at two representative disturbance frequencies, based on a discrete Fourier transform of the computed data at $\mathrm{f}=240 \mathrm{kHz}$ and $400 \mathrm{kHz}$, respectively, are plotted in Figs. 5(a) and 5(b). Because of the strong destabilizing influence of stationary crossflow vortices, the azimuthal region of the highest amplitudes of secondary disturbances in Fig. 5(a) corresponds to the region of the crossflow vortices (centered near $\phi=135 \mathrm{deg}$. near the outflow boundary). Figure 5(a) also shows a second peak with weaker fluctuations, which is located near $\phi=160$ degrees at $X=0.40 \mathrm{~m}$. There are no significant crossflow vortices in this region and the secondary peak is associated with purely Mack mode instabilities of the underlying boundary layer over the cone. At the higher disturbance frequency of $400 \mathrm{kHz}$, the azimuthal region of the Mack mode fluctuations shifts toward the windward meridian. Overall, the highest amplification factors over the entire cone surface correspond to a disturbance frequency of $240 \mathrm{kHz}$. 


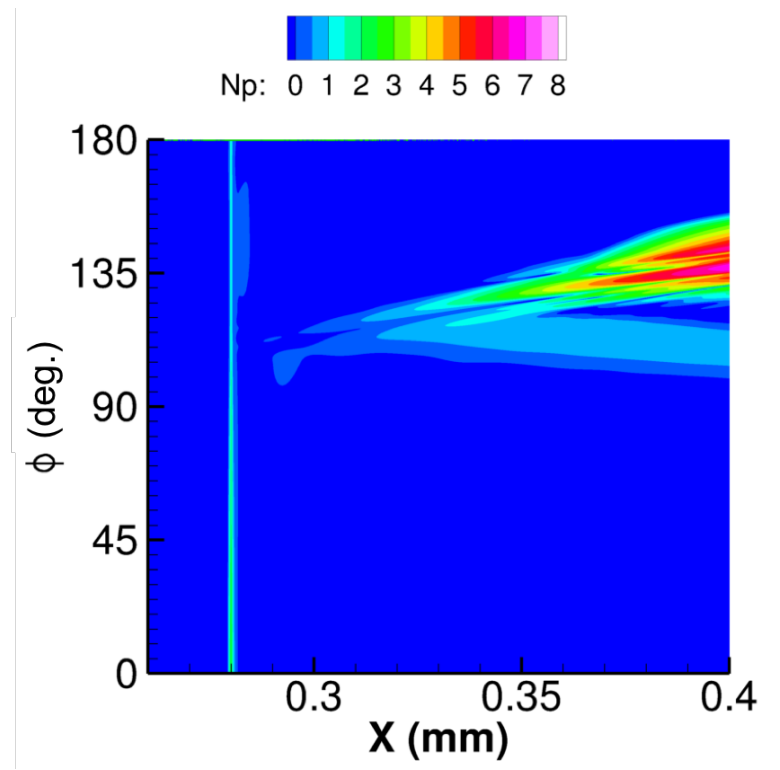

(a) $f=240 \mathrm{kHz}$.

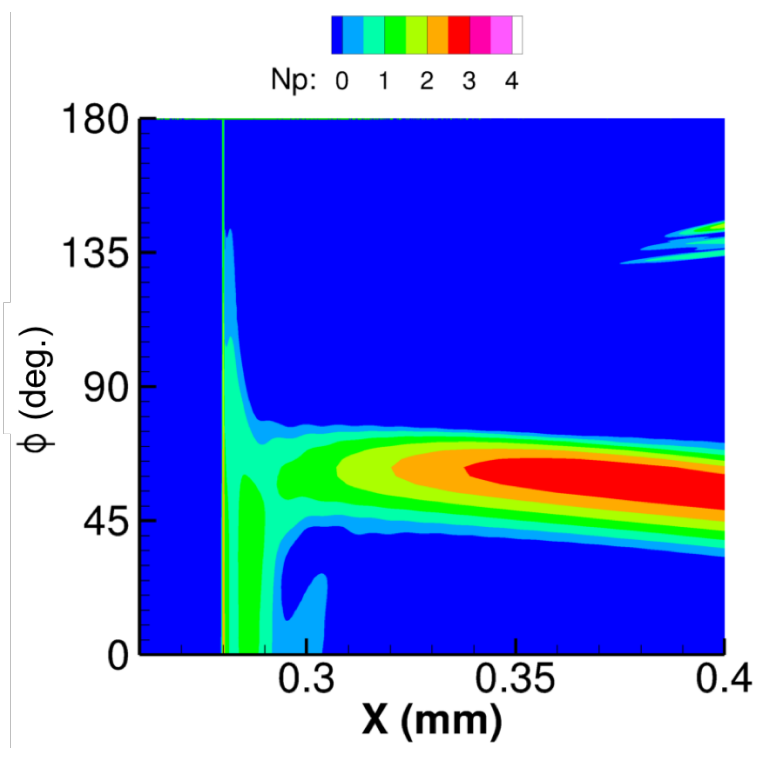

(b) $f=400 \mathrm{kHz}$.

Fig. 5. DNS results for $\left|p^{\prime}\right|$ contours on cone surface for selected forcing frequencies. ${ }^{48}$

First, we note that the PSE mode shapes for the $\left|u^{\prime}\right|$ fluctuations due to mode MMV1 (Fig. 3(a)) are very similar to the DNS results from Fig. 9(a) of Ref. [48]. Indeed, the PSE predictions are also successful in capturing a rather subtle feature of the mode shapes from the DNS results. Specifically, we note that the MMV1 mode shapes in Fig. 3(a) indicate a switchover in the location of peak $\left|u^{\prime}\right|$ fluctuations from the shear layer of vortex V1 at $X=0.364 \mathrm{~m}$ to the shear layer of vortex V2 at $X=0.385 \mathrm{~m}$, and then back to the vortex V1 at $X=0.405 \mathrm{~m}$. An identical behavior was also observed in the DNS calculations, suggesting that the mode MMV1 may account for a dominant portion of the disturbance field from the DNS solution.

Mode shapes of $\left|p^{\prime}\right|$ perturbations at selected locations from the DNS flowfield and the corresponding PSE predictions for mode MMV1 at the same locations are shown in Figs. 6(a) and 6(b), respectively. The DNS mode shape at $X=0.313 \mathrm{~m}$ indicates two separate azimuthal regions of high amplitude fluctuations. The windward peak corresponds to Mack mode fluctuations inboard of the crossflow vortex pattern, whereas the other peak corresponds to the secondary instability modes of the crossflow vortex pattern as seen from the surface pressure fluctuations in Fig. 5(a). The inboard fluctuations are excited as Mack mode instabilities of the boundary layer flow without crossflow vortices. The wall forcing in DNS extends across the entire circumference of the cone and hence, can excite instability modes of the boundary layer regions with and without the crossflow vortices. However, the instabilities modes of the boundary layer flow outside of the crossflow vortex packet are neither targeted nor expected to be captured within the PSE predictions. Between $X=0.330 \mathrm{~m}$ and $X=0.385 \mathrm{~m}$, the peak pressure fluctuations in DNS are located close to the surface underneath vortex V1, similar to the PSE predictions for mode MMV1 in Fig. 6(b). As a result of the continued interaction with the underlying crossflow vortex, the peak pressure fluctuations at $X=0.405 \mathrm{~m}$ have shifted away from the surface and to the top of the crossflow vortex structure. The same trend is also captured by the MMV1 mode predictions based on PSE. The DNS solution also indicates relatively stronger $\left|p^{\prime}\right|$ fluctuations within vortex V2 for $X \geq 0.385 \mathrm{~m}$, and that feature is again predicted by the PSE.

Frequency spectra of the disturbance N-factor from the DNS calculations and those of mode MMV1 from the PSE computations are shown in Figs. 7(a) and 7(b), respectively. Both $\mathrm{N}$-factors are based on the integral of the disturbance kinetic energy across the cross-section of the vortex pattern. The PSE N-factors are defined with respect to the neutral location. However, because the neutral station cannot be easily defined from the DNS calculation due to its proximity to the wall forcing, the DNS N-factors are shifted vertically so that the $\mathrm{N}$-factor for $f=240 \mathrm{kHz}$ matches the PSE prediction. We observe that the peak of the N-factor spectra corresponds to nearly the same frequency in both DNS and PSE calculations. The peak frequency from the DNS results corresponds to $f=240$ $\mathrm{kHz}$, subject to some uncertainty due to the relatively coarse frequency resolution of the DNS analysis $(\Delta f \approx 40$ 
$\mathrm{kHz}$ ). The peak frequency from the PSE calculations is slightly higher, between $f=240 \mathrm{kHz}$ and $f=260 \mathrm{kHz}$, depending on the axial location; however, the discrepancy in peak frequency could, in part, be caused by the finer resolution of the PSE calculations ( $\Delta f \approx 20 \mathrm{kHz}$ ).

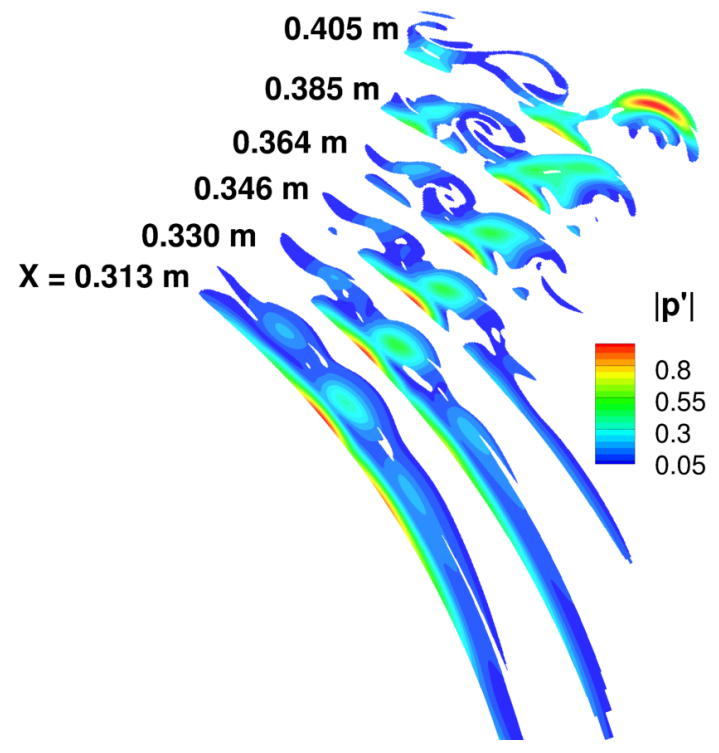

(a) DNS.

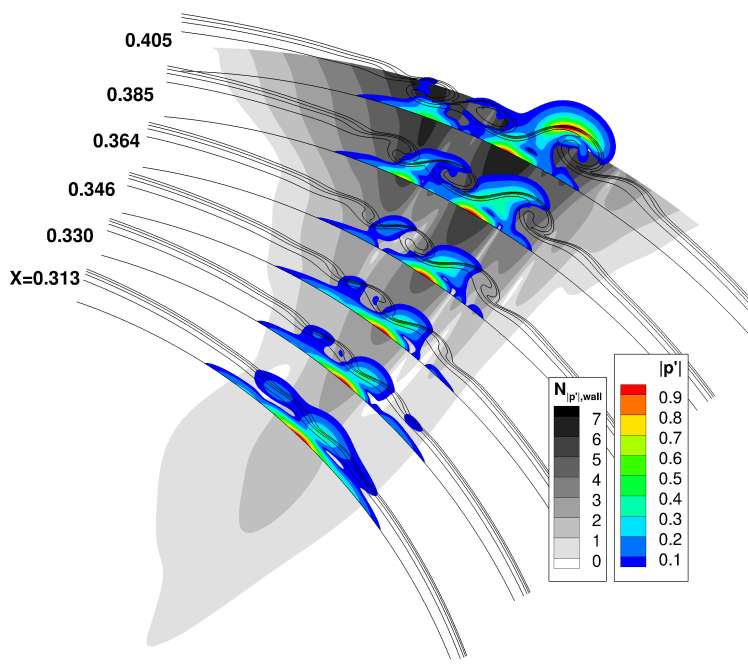

(b) Plane marching PSE (mode MMV1).

Fig. 6. Contours of disturbance amplitudes ("mode shapes") based on pressure fluctuations at $f=$ $240 \mathrm{kHz}$ and selected axial stations. At each station, the peak fluctuation amplitude is normalized to unity. To highlight the regions of strong fluctuations at multiple axial stations, contours are shown only within the boundary layer region.

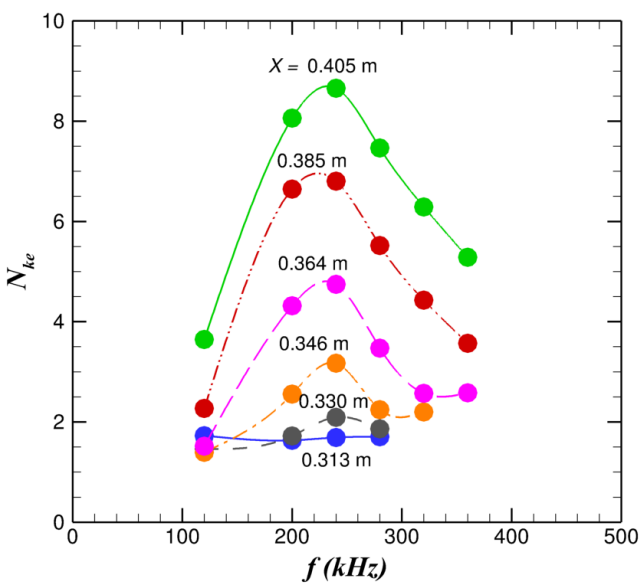

(a) DNS.

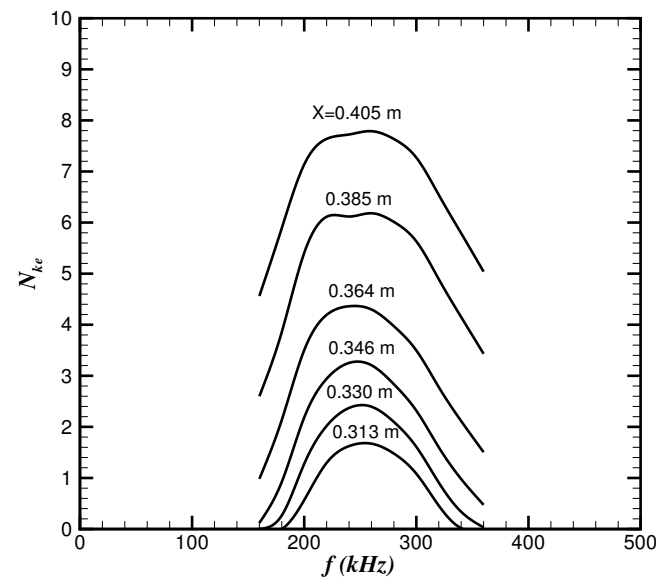

(b) Plane marching PSE (MMV1 mode).

Fig. 7. N-factor spectra based on peak surface pressure fluctuation at selected axial stations. To focus on the effects of the compact crossflow pattern, fluctuations at azimuthal locations beyond the crossflow pattern are ignored in this analysis. 
Figure 8 shows the $\mathrm{N}$-factor evolution for high-frequency instabilities with $f=240 \mathrm{kHz}$ as computed via the DNS, plane marching PSE, and the quasiparallel predictions. Over a significant range of distances, the PSE based $\mathrm{N}$-factor curves for modes MMV1 and MMV2 are rather close to each other. To begin with, the $\mathrm{N}$-factor for the MMV2 mode is slightly less than that of the MMV1 mode. However, near $X \approx 0.36$, the MMV2 N-factor crosses above the $\mathrm{N}$-factor curve for the MMV1 mode. A notable difference between the evolution of these two modes involves their far downstream behavior near $X=0.4$, where the MMV2 mode ceases to amplify whereas the MMV1 mode continues to grow across the entire region included in the basic state calculation for the crossflow vortex pattern. The domain for the DNS from Ref. [48] continues up to $X=0.41$ and the continued rise of the $\mathrm{N}$-factor in the DNS solution is analogous to the MMV1 mode from the PSE predictions. This behavior is consistent with the mode shape similarity between the DNS solution and the MMV1 mode, and tends to confirm that the DNS solution is dominated by the MMV1 mode.

Figure 8 also shows that the $\mathrm{N}$-factors based on the PSE predictions are in relatively good agreement with those inferred from the DNS solution, whereas the N-factors based on quasiparallel predictions are substantially below the PSE and DNS calculations. The difference in PSE and quasiparallel N-factors for the MMV1 mode near $X=0.41$ is approximately $\Delta \mathrm{N} \approx 3.5$, whereas the quasiparallel $\mathrm{N}$-factor for the MMV2 is way below the $\mathrm{N}$-factor values from both the PSE and DNS predictions; it reaches a peak value of less than 2 near $X=0.37$ and decays rapidly beyond that location.

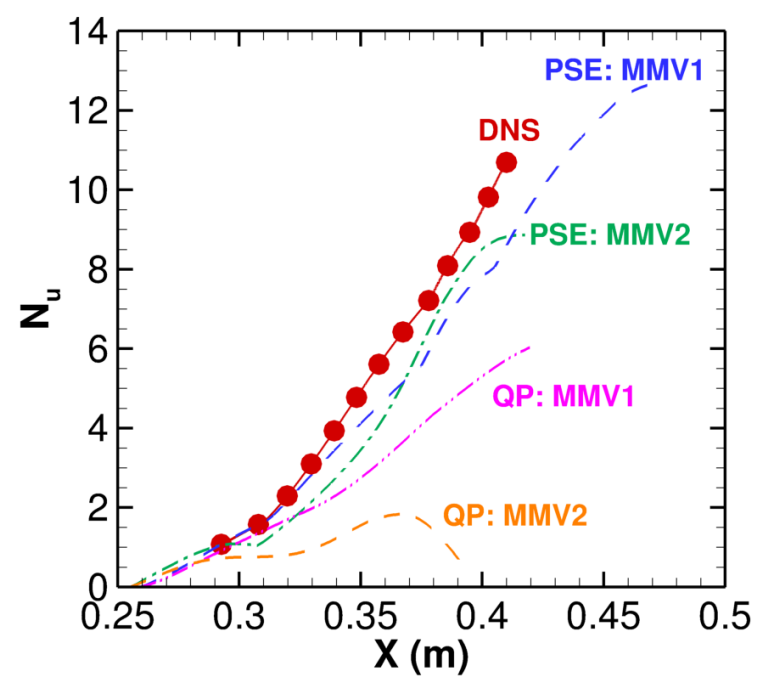

Fig. 8. N-factor evolution for $f=240 \mathrm{kHz}$.

\section{Nonlinear Development of Secondary Instabilities}

New DNS calculations were performed to investigate the nonlinear propagation of high frequency instabilities in the presence of the same azimuthally localized vortex pattern due to single wavelength forcing as described earlier in relation to Fig. 1(d). The results of Section IV showed that the PSE predictions for mode MMV1 agreed fairly well with the DNS solution of Choudhari et al. ${ }^{48}$ corresponding to sufficiently low amplitude forcing at the wall. The DNS computations described in the present section examine the effects of nonlinearity on the propagation of mode MMV1, which is introduced via inflow forcing based on the mode shape predicted by the plane marching PSE. The amplitude of the inflow forcing is increased systematically to study the nonlinear disturbance field created by the self-interaction of a single frequency mode at $f=240 \mathrm{kHz}$. The spatial grid used in this case was identical to that used in Ref. [48] for the linear simulation from Fig. 5 above.

$\mathrm{N}$-factor evolution based on the peak velocity fluctuation for initial amplitudes ranging from $1 \times 10^{-6}$ to $5 \times 10^{-3}$ is shown in Fig. 9. As the fluctuation amplitude becomes sufficiently large at downstream locations, nonlinear effects reduce the disturbance growth rates and the amplitude evolves in a quasisaturated manner within the aft portion of the domain. As the inflow amplitude increases, the onset of quasisaturation shifts to progressively 
upstream lcoations. Although not shown, the peak modal amplitude in the quasisaturated region falls between 15 to 20 percent of the tunnel freestream velocity.

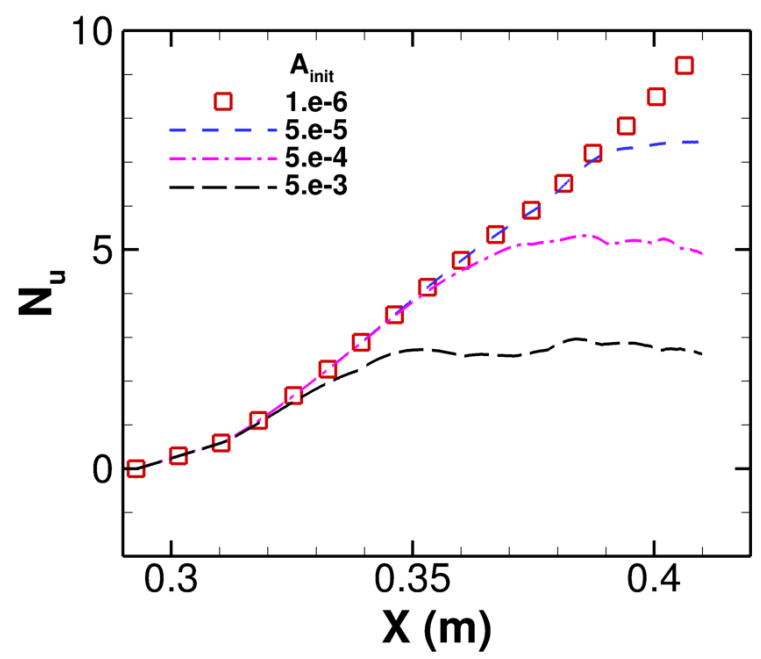

Fig. 9. N-factor evolution for mode MMV1 for different initial amplitudes $(f=240 \mathrm{kHz})$.

We next examine additional features of the DNS solution for the case of the largest inflow amplitude from Fig. 9. Contours of time averaged wall shear near the cone surface, along with crossplane contours of r.m.s. fluctuation in the axial velocity at $X=0.32 \mathrm{~m}$ and $X=0.40 \mathrm{~m}$ are shown in Figs. 10(a) and 10(b), respectively. For easier interpretation, the wall shear values are normalized to a peak value of unity below vortex $\mathrm{V} 1$ at $X=0.313 \mathrm{~m}$. The r.m.s. fluctuation contours at the upstream station (i.e., $X=0.32 \mathrm{~m}$ ) resemble the linear mode shape predicted by the plane marching PSE (Fig. 3(a)). At this early stage, the high-frequency secondary instabilities are not accompanied by notable changes in the mean wall shear. However, as those instability waves amplify along the downstream region, the associated Reynolds stresses eventually cause the mean wall shear to rise, as indicated by the small, triangular region of high mean shear just upstream of the location of $u_{r m s}$ contours at $X=0.40 \mathrm{~m}$ (Fig. 10(b)). Although not shown, the mean wall shear begins to rise near $X=0.39 \mathrm{~m}$ and eventually reaches a value of nearly 5 times the peak wall shear within the upstream region.

The rapid rise in skin friction is symptomatic of the onset of laminar-turbulent transition, which first occurs near the apex of the approximately wedge shaped region of high wall shear within the surface contours from Fig. 10(b). The lateral spreading of high-shear region along the wedge is analogous to that observed previously during the breakdown of secondary instability from the DNS of a subsonic boundary layer on a swept airfoil. ${ }^{59,60}$ The half $^{\text {The }}$ angle associated with the turbulent wedge is approximately 4 degrees, which is very close to the upper bound of the measured range of turbulence spreading angles for a boundary layer edge Mach number of 6, as reported by Fischer. ${ }^{66}$ Even though the MMV1 mode has a hybrid character consisting of Mack modes that peak near the wall and SI modes that peak away from the wall (Fig. 9(a)), the MMV1 mode shape within the downstream part of the computational domain is closer to that of the SI modes (Fig. 3(a)). The latter trend could account for the similarity between the wall shear evolution in the present case with the transition zone within a subsonic, swept wing boundary layer. ${ }^{59,60}$ Longer domain simulations with higher spatial resolution must be performed to capture the full details of the transitional flow including the fully developed turbulent boundary layer. Our follow-on effort is focused on those simulations. 


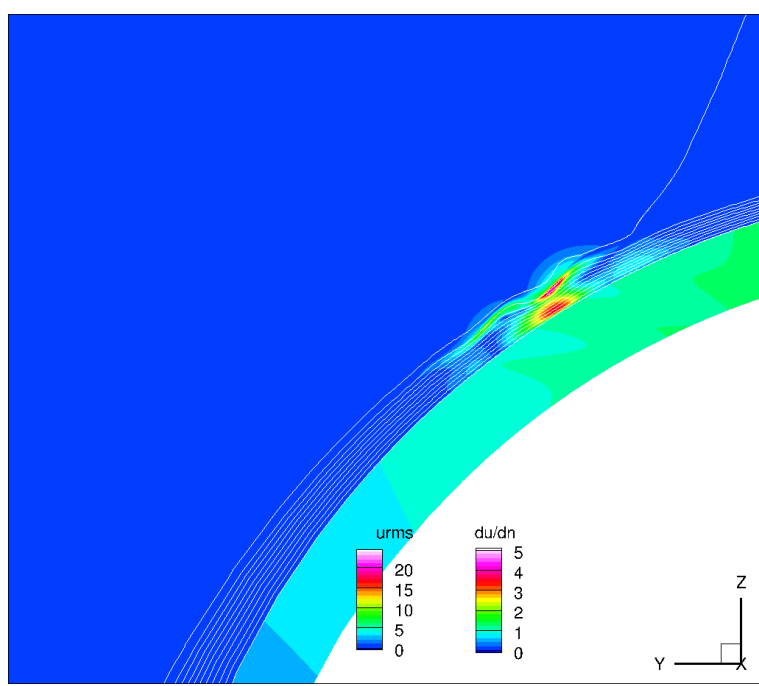

(a) $X=0.32 \mathrm{~m}$.

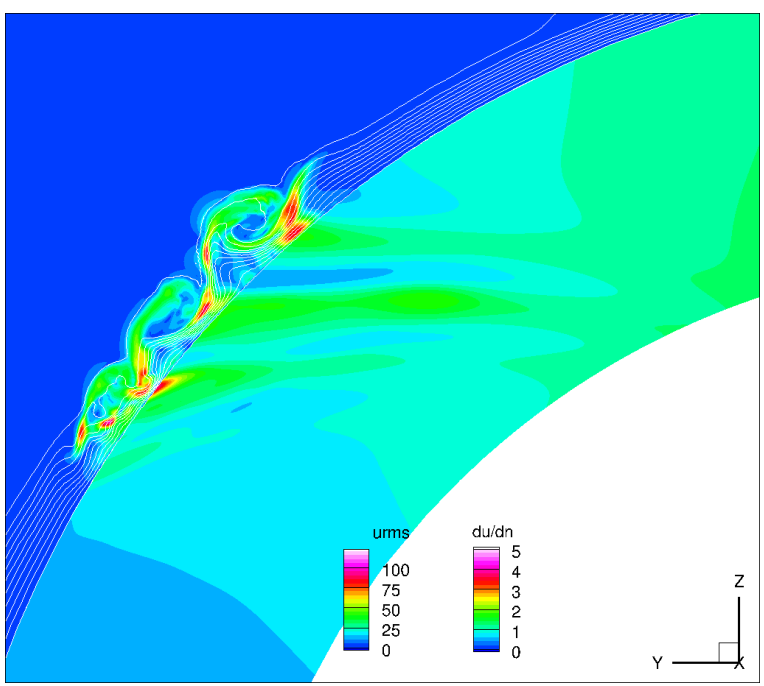

(b) $X=0.400 \mathrm{~m}$

Fig. 10. Contours of time averaged wall shear near the cone surface, along with crossplane contours of $u_{r m s}$ at selected $x$ locations. White line contours superposed on $u_{r m s}$ contours denote the time averaged axial velocity contours within the crossplane.

\section{Summary and Concluding Remarks}

Previously performed DNS of stationary crossflow instability of azimuthally periodic ${ }^{31}$ and azimuthally compact $^{48}$ crossflow vortex patterns and their high-frequency secondary instabilities have been extended to include intermediate azimuthal widths of the vortex pattern, to bridge the gap between the two opposite limiting cases from the previous papers. Furthermore, linear stability analysis for a compact crossflow pattern is extended to include nonparallel predictions and a quantitative comparison with the DNS results. Results show that the quasiparallel predictions can reveal the general topology of instability modes and, to some extent, their approximate spatiotemporal characteristics. However, they do not provide accurate predictions of instability growth in the present case, and lead to severe underprediction of the $\mathrm{N}$-factors for the dominant instability modes. In contrast, the nonparallel framework is able to yield predictions that agree more closely with the DNS results and the agreement between the two extends across mode shapes, dominant frequencies, as well as the $\mathrm{N}$-factors of the most amplified instability modes. The PSE results also capture relatively subtle features of the DNS solutions in terms of a switchover in peak disturbance location from one vortex to another, and back shortly thereafter. However, the agreement between the DNS and the PSE is not perfect and further work is necessary to identify the cause for those smaller discrepancies.

Furthermore, the effects of disturbance nonlinearity on the instability modes of a compact vortex pattern, including the initial phase of laminar-turbulent transition due to MMV1 mode of instability have been investigated via DNS for the first time. Results of these simulations indicate certain common features with the previous computations of crossflow transition over subsonic swept wings.

\section{Acknowledgments}

This work was performed as part of the Revolutionary Computational Aerosciences discipline under the Transformational Tools and Technologies project of the NASA Transformative Aeronautics Concepts Program. Computational resources for this work were provided by the NASA High-End Computing (HEC) Program through the NASA Advanced Supercomputing (NAS) Division at Ames Research Center. MC would like to thank Prof. Steven Schneider and Mr. Joshua Edelman from Purdue University for several technical discussions. The authors also acknowledge Prof. Pino Martin from the University of Maryland for providing the original version of the DNS code used in this effort. 


\section{References}

1 Mack, L. M., Boundary-Layer Linear Stability Theory, in "Special Course on Stability and Transition of Laminar Flow," AGARD Report No. 709, pp. 3.1-3.81, 1984.

2 Li, F., Choudhari, M. M., Chang, C.-L., Wu, M. and Greene, P. T., "Development and Breakdown of Görtler Vortices in High Speed Boundary Layers,” AIAA Paper 2010-705, 2010.

3 Saric, W. S., Reed, H. L., and White, E. B., "Stability and Transition of Three-Dimensional Boundary Layers," Ann. Rev. Fluid Mech., Vol. 35, 2003, pp. 413-440.

4 Dougherty, N. S. and Fisher, D. F., "Boundary Layer Transition on a 10-degree Cone: Wind Tunnel/Flight Data Correlation," AIAA Paper 80-0154, 1980.

5 King, R. A., "Three-Dimensional Boundary-Layer Transition on a Cone at Mach 3.5," Experiments in Fluids, Vol. 13, No. 5, 1992, pp. 305-314.

6 Malik, M. R. and Balakumar, P., "Instability and Transition in Three-Dimensional Supersonic Boundary Layers," AIAA Paper 1992-5049, 1992.

7 Hanifi, A. and Dahlkild, A. A., "Some Stability Characteristics of the Boundary Layer on a Yawed Cone," AIAA Paper 19933048, 1993.

8 Sugiura, H., Tokugawa, N., Nishizawa, A., Ueda, Y., Ishikawa, H., and Yoshida, K., "Boundary-Layer Transition on Axisymmetric Bodies with Angles of Attack in Supersonic Flow," Proceedings of 2003 Annual Meeting, Japan Society of Fluid Mechanics, (in Japanese), 2003, pp. 352-353.

9 Balakumar, P., "Stability of Supersonic Boundary Layers on a Cone at an Angle of Attack," AIAA Paper 2009-3555, 2009.

${ }^{10}$ Tokugawa, N., Choudhari, M., Ishikawa, H., Ueda, Y., Fujii, K., Atobe, T., Li, F., Chang, C.-L., and White, J., "Transition Along Leeward Ray of Axisymmetric Bodies at Incidence in Supersonic Flow," AIAA Journal, Vol. 53, No. 12, 2015 , pp. 3737-3751. doi: 10.2514/1.J054070

${ }^{11}$ Choudhari, M., Tokugawa, N., Li, F., Chang, C.-L., White, J., Ishikawa, H., Ueda, Y., Atobe, T., and Fujii, K., "Computational Investigation of Supersonic Boundary Layer Transition over Canonical Fuselage Nose Configurations," Proc. of $7^{\text {th }}$ Intl. Conference on Computational Fluid Dynamics, Island of Hawaii, June 2012 (http://www.iccfd.org/iccfd7/assets/pdf/papers/ICCFD7-2306 paper.pdf).

12 Schuele, C. Y., Corke, T. C., and Matlis, E., "Control of Stationary Crossflow Modes in a Mach 3.5 Boundary Layer Using Patterned Passive and Active Roughness," Journal of Fluid Mechanics, Vol. 718, 2013, pp. 5-38.

${ }^{13}$ Stetson, K. F., Thompson, E. R., Donaldson, J. C., and Siler, L. G., "Laminar Boundary Layer Stability Experiments on a Cone at Mach 8 - Part 3: Sharp Cone at Angle of Attack," AIAA Paper 85-0492, 1985.

${ }^{14}$ Schneider, S. P., "Hypersonic Laminar-Turbulent Transition on Circular Cones and Scramjet Forebodies," Prog. in Aerospace Sciences, Vol. 40, 2004, pp. 1-5.

${ }^{15}$ Swanson, E., "Boundary-Layer Transition on Cones at Angle of Attack in a Mach 6 Quiet Tunnel," Ph.D. Dissertation, School of Aeronautics and Astronautics, Purdue University, 2008.

${ }^{16}$ Swanson, E. and Schneider, S. P., "Boundary-Layer Transition on Cones at Angle of Attack in a Mach-6 Quiet Tunnel," AIAA Paper 2010-1062, 2010.

${ }^{17}$ Li, F., Choudhari, M., Chang, C.-L., and White, J., “Analysis of Instabilities in Non-Axisymmetric Hypersonic Boundary Layers over Cones," AIAA Paper 2010-4643, 2010.

${ }^{18}$ Balakumar, P. and Owens, L., "Stability of Hypersonic Boundary Layers on a Cone at Angle of Attack," AIAA Paper 20104718, 2010.

${ }^{19}$ Li, F., Choudhari M., Chang, C.-L., White, J. A., Kimmel, R., Adamczac, D., Borg, M., Stanfeld, S., and Smith, M., "Stability Analysis for HIFiRE Experiments," AIAA Paper 2012-2961, 2012.

${ }^{20}$ Munoz, F., Heitmann, D., and Radespiel, R., "Instability Modes in Boundary Layers of an Inclined Cone at Mach 6," J. Spacecraft and Rockets, Vol. 51, No. 2, 2014, pp. 442-454.

${ }^{21}$ Ward, C. A. C., Henderson, R. O., and Schneider, S. P., "Possible Secondary Instability of Stationary Crossflow Vortices on an Inclined Cone at Mach 6," AIAA Paper 2015-2773, 2015.

${ }^{22}$ McKiernan, G. R., Chynoweth, G. R., Edelman, J. B., McKenzie, J. A., and Sweeney, C. J., "Instability and Transition Experiments in the Boeing/AFOSR Mach 6 Quiet Tunnel," AIAA Paper 2015-2317, 2015.

${ }^{23}$ Edelman J. and Schneider, S. P., "New Measurements of the Secondary Instability of Stationary Crossflow Waves in the Mach-6 Quiet Tunnel," NATO-STO AVT-240 \& RTG-082 Meeting on Hypersonic Boundary Layer Transition, Prague, Czech Republic, Oct. 15-16, 2015.

${ }^{24}$ Craig S. A. and Saric W, "Crossflow Instability on a Yawed Cone at Mach 6," 8th IUTAM Symposium on LaminarTurbulent Transition, Procedia IUTAM, Vol. 14, Medeiros. M. A. F. and Meneghini, J. R. (eds.), 2015, pp. 15-25.

${ }^{25}$ Craig, S. A. and Saric, W. S., "Experimental Study of Crossflow Instability on a Mach 6 Yawed Cone," AIAA Paper 2015$2774,2015$.

${ }^{26}$ Oliviero, N. B., Kocian, T. S., Moyes, A. J., and Reed, H. L., "EPIC: NPSE Analysis of Hypersonic Crossflow Instability on Yawed Straight Circular Cone," AIAA Paper 2015-2772, 2015.

${ }^{27}$ Stanfield, S., Kimmel, R. A., Adamczac, D., and Juliano, T., "Boundary-Layer Transition Experiment During Reentry of HIFiRE-1,” J. Spacecraft and Rockets, Vol. 52, 2015, pp. 637-649.

${ }^{28}$ Juliano, T., Kimmel, R. A., Willems, S., Guelhan, A., and Wagnild, R., "HIFiRE-1 Boundary-Layer Transition: Ground Test Results and Stability Analysis,” AIAA Paper 2015-1736, 2015. 
${ }^{29}$ Li, F., Choudhari, M., and Paredes, P., "Nonlinear Evolution and Secondary Instability of Crossflow Disturbances in Hypersonic Boundary Layer over a Cone," NATO-STO AVT-240 \& RTG-082 Meeting on Hypersonic Boundary Layer Transition, Prague, Czech Republic, Oct. 15-16, 2015.

${ }^{30}$ Li, F., Choudhari, M., Paredes, P., and Duan, L., "Secondary Instability of Stationary Crossflow Vortices in Mach 6 Boundary Layer over a Circular Cone,” NASA/TM-2015-218997, Dec. 2015.

${ }^{31}$ Li, F., Choudhari, M., Paredes, P., and Duan, L., "High-Frequency Instabilities of Stationary Crossflow Vortices in a Hypersonic Boundary Layer," Phys. Rev. Fluids, Vol. 1, 2016, 053603.

32 Moyes, A. J., Paredes, P., Kocian T. S., and Reed, H., "Secondary Instability Analysis of Crossflow on a Hypersonic Yawed Straight Circular Cylinder," AIAA Paper 2016-0848, 2016.

${ }^{33}$ Kimmel, R., "Laminar-Turbulent Transition in a Mach 8 Elliptic Cone Flow," AIAA J., Vol. 37, No. 9, 1999, pp. $1080-1087$.

${ }^{34}$ Poggie, J. and Kimmel, R., "Traveling Instability Waves in a Mach 8 Flow over an Elliptic Cone,” AIAA J., Vol. 38, No. 2, 2000 , pp. 251-258.

${ }^{35}$ Kimmel, R. L., and Poggie, J., "Transition on an Elliptic Cone at Mach 8," American Society of Mechanical Engineers ASME FEDSM97-3111, June 1997.

${ }^{36}$ Kimmel, R. L., Poggie, J. and Schwoerke, S. N., "Laminar-Turbulent Transition in a Mach 8 Elliptic Cone Flow," AIAA Journal, Vol. 37, No. 9, 1999, pp. 1080-1087.

${ }^{37}$ Holden, M., "Experimental Studies of Laminar, Transitional, and Turbulent Hypersonic Flows Over Elliptic Cones at Angle of Attack," Air Force Office of Scientific Research Technical Report AFRL-SR-BL-TR-98-0142, Bolling Air Force Base, DC, 1998.

${ }^{38}$ Schmisseur, J. D., Schneider, S. P., and Collicott, S. H., "Response of the Mach 4 Boundary Layer on an Elliptic Cone to Laser-Generated Freestream Perturbations," AIAA Paper 1999-0410, 1999.

${ }^{39}$ Choudhari, M., Chang, C.-L., Li, F., Edwards, J., and Candler, G., "Hypersonic Boundary Layer Transition: Roughness Effects and 3D Configurations," NASA Fundamental Aeronautics Program Annual Meeting, Atlanta, GA, October 5-7, 2008.

${ }^{40}$ Berger, K., Rufer, S., Kimmel, R., and Adamczak, D., "Aerothermodynamic Characteristics of Boundary Layer Transition and Trip Effectiveness of the HIFiRE Flight 5 Vehicle," AIAA Paper 2009-4055, 2009.

${ }^{41}$ Choudhari, M., Chang, C.-L., Li, F., Berger, K., Candler, G., and Kimmel, R., "Transition Analysis for the HIFiRE-5 Vehicle," AIAA Paper 2009-4056, 2009.

${ }^{42}$ Berger, K., Rufer, S., Kimmel, R., and Adamczak, D., "Aerothermodynamic Characteristics of Boundary Layer Transition and Trip Effectiveness of the HIFiRE Flight 5 Vehicle," AIAA Paper 2009-4055, 2009.

${ }^{43}$ Kimmel, R. A., Adamczac, D., and Juliano, T., "HIFiRE-5 Flight Test Preliminary Results,” AIAA Paper 2013-0377, 2013.

${ }^{44}$ Paredes, P. and Theofilis, V., "Centerline Instabilities on the Hypersonic International Flight Research Experimentation HIFiRE-5 Elliptic Cone Model,” Journal of Fluids and Structures, Vol. 53, 2015, pp. 36-49.

45 Juliano, T., Adamczac, D., and Kimmel, R. A., "HIFiRE-5 Flight Test Results," J. Spacecraft and Rockets, Vol. 52, 2015, pp. $650-663$.

${ }^{46}$ Juliano, T., Borg, M., and Schneider, S. P., "Quiet Tunnel Measurements of HIFiRE-5 Boundary-Layer Transition," AIAA Journal, Vol. 53, 2015, pp. 832-84.

${ }^{47}$ Borg, M., Kimmel, R. A., and Stanfield, S., “Traveling Crossflow Instability for the HIFiRE-5 Elliptic Cone,” J. Spacecraft and Rockets, Vol. 52, 2015, pp. 664-673.

${ }^{48}$ Choudhari, M., Li, F., Paredes, P., and Duan, L., "Computations of Crossflow Instability in Hypersonic Boundary Layers," AIAA Paper 2017-4300, 2017.

${ }^{49}$ vulcan-cfd.larc.nasa.gov (last accessed October 17, 2016)

50 van Albada, G. D., van Leer, B., and Roberts, W. W., "A Comparative Study of Computational Methods in Cosmic Gas Dynamics," Astronomy and Astrophysics, Vol. 108, 1982, pp. 76-84.

${ }^{51}$ Edwards, J. R., “A Low-Diffusion Flux-Splitting Scheme for Navier-Stokes Calculations,” Computer and Fluids, Vol. 6, 1997, pp. 635-659.

${ }^{52}$ Litton, D., Edwards, J., and White, J., “Algorithmic Enhancements to the VULCAN Navier-Stokes Solver," AIAA Paper 2003-3979, 2003.

${ }^{53}$ Cheatwood, F. M. and Gnoffo, P. A., "User's Manual for the Langley Aerothermodynamic Upwind Relaxation Algorithm (LAURA),"NASA TP 4674, April 1996.

${ }^{54} \mathrm{Wu}$, M. and Martin, M. P., "Direct Numerical Simulation of Supersonic Boundary Layer over a Compression Ramp," AIAA Journal, Vol. 45, No. 4, 2007, pp. 879-889.

55 Jiang, G. S. and Shu, C. W., "Efficient Implementation of Weighted ENO Schemes," J. Comp. Phys., Vol. 126, No. 1, 1996, pp. 202-228.

56 Taylor, E. M., Wu, M., and Martin, M. P., "Optimization of Nonlinear Error Sources for Weighted Non-Oscillatory Methods in Direct Numerical Simulations of Compressible Turbulence," J. Comp. Phys., Vol. 223, No. 1, 2006, pp. 384-397.

${ }^{57}$ Williamson, J., "Low-Storage Runge-Kutta Schemes," J. Comp. Phys., Vol. 35, No. 1, 1980, pp. 48-56.

${ }^{58}$ Duan, L., Beekman, I., and Martin, M. P., "Direct Numerical Simulation of Hypersonic Turbulent Boundary Layers. Part 3: Effect of Mach Number," J. Fluid. Mech., Vol. 672, 2011, pp. 245-267.

${ }^{59}$ Duan, L., Choudhari, M., and Li, F., "Direct Numerical Simulation of Crossflow-Induced Transition in a Swept Wing Boundary Layer,” AIAA Paper 2013-2617, 2013. 
${ }^{60}$ Choudhari, M., Li, F., Duan, L., Carpenter, M. H., Streett, C. L., and Malik, M. R., "Towards Bridging the Gaps in Holistic Transition Prediction via Numerical Simulations," AIAA Paper 2013-2718, 2013.

${ }^{61} \mathrm{Li}$, F. and Choudhari, M., "Spatially Developing Secondary Instabilities and Attachment Line Instability in Supersonic Boundary Layers," AIAA Paper 2008-590, 2008.

${ }^{62}$ Li, F. and Choudhari, M., "Spatially Developing Secondary Instabilities in Compressible Swept Airfoil Boundary Layers," Theoretical and Computational Fluid Dynamics, Vol. 25, June 2011, pp. 65-84.

${ }^{63}$ Paredes, P., Hanifi, A., Theofilis, V., and Henningson, D., "The Nonlinear PSE-3D Concept for Transition Prediction in Flows with a Single Slowly-Varying Spatial Direction," Procedia IUTAM, Vol. 14C, 2015, pp. 35-44.

${ }^{64}$ De Tullio, N., Paredes, P., Sandham, N., and Theofilis, V., "Roughness-Induced Instability and Breakdown to Turbulence in a Supersonic Boundary-Layer," J. Fluid Mech., Vol. 735, 2013, pp. 613-646.

${ }^{65}$ Choudhari, M., Chang, C.-L., and Jiang, L., "Towards Transition Modeling for Supersonic Laminar Flow Control," Philosophical Transactions of Royal Society of London (Physical and Mathematical Sciences), Vol. 363, No. 1830, May 2005, pp. 1079-1096.

${ }^{66}$ Fischer, M. C., "Spreading of a Turbulent Disturbance,” AIAA Journal, Vol. 10, No. 7, 1972, pp. 957-959. 